\title{
Radio Loud AGN in the 2XMMi catalogue
}

\author{
Alvaro Labiano ${ }^{1}$, Matteo Guainazzi ${ }^{1}$, and Stefano Bianchi ${ }^{2}$ \\ ${ }^{1}$ European Space Astronomy Centre of ESA, Madrid, Spain \\ email: Alvaro.Labiano@esa.int, Matteo.Guainazzi@esa.int \\ ${ }^{2}$ Dipartimento di Fisica, Università degli Studi Roma Tre, Rome, Italy \\ email: bianchi@fis.uniroma3.it
}

\begin{abstract}
We are carrying out a search for all radio loud Active Galactic Nuclei observed with $X M M$-Newton, including targeted and field sources to perform a multi-wavelength study of these objects. We have cross-correlated the Verón-Cetty \& Verón (2010) catalogue with the $X M M$ Newton Serendipitous Source Catalogue (2XMMi) and found around 4000 sources. A literature search provided radio, optical, and X-ray data for 403 sources. This poster summarizes the first results of our study.
\end{abstract}

Keywords. Galaxies: active - Galaxies: Seyfert - quasars: general - X-rays: general

\section{Introduction and sample}

Bianchi et al. (2009a,b) presented the Catalogue of Active Galactic Nuclei (AGN) in the XMM-Newton Archive (CAIXA). They focused on the radio-quiet, X-ray unobscured $\left(\mathrm{NH}<2 \times 10^{22} \mathrm{~cm}^{-2}\right)$ AGN observed by XMM-Newton in targeted observations. We are carrying out a similar multiwavelength study, for both targeted and field radio-loud AGN observed by XMM Newton. We cross-correlated the Verón-Cetty \& Verón (2010) catalogue (Quasars and Active Galactic Nuclei, 13th edition) with the XMM-Newton Serendipitous Source Catalogue (2XMMi, Watson et al. 2009) Third Data Release, and obtained a list of around 4000 sources. However, only $10 \%$ of the sources have published

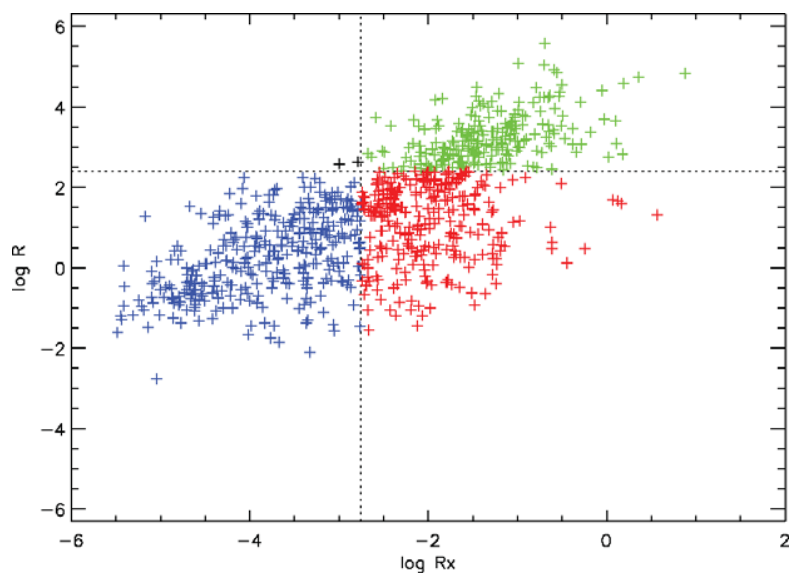

Figure 1. Radio loud (green) and radio quiet (blue and red) AGN. Rx is the ratio between 5 $\mathrm{GHz}$ and $2-10 \mathrm{keV}$ emission. $\mathrm{R}$ is the ratio between $5 \mathrm{GHz}$ and $\mathrm{B}$-band emission. Boundares from Panessa et al. 2007. Blue points are sources classified as radio quiet by $\mathrm{R}$ and $\mathrm{Rx}$ parameters Red points are sources classified as radio quiet by the $\mathrm{R}$ parameter and radio loud by the $\mathrm{Rx}$ parameter 


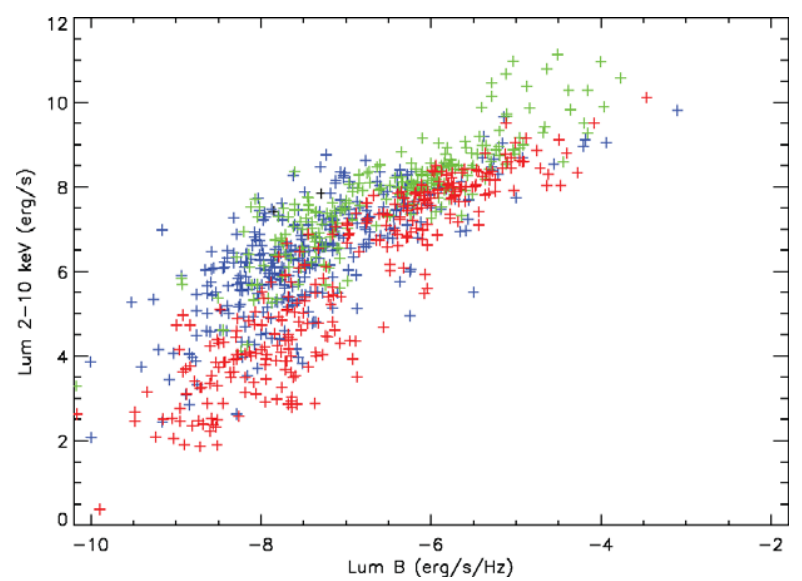

Figure 2. X-ray versus B-band luminosities. For optical luminosities higher than $10^{-6} \mathrm{erg} / \mathrm{s} / \mathrm{Hz}$ radio loud (green) AGN are brighter in $\mathrm{X}$-rays than radio quiet (red and blue). This effect is stronger at higher luminosities $\left(10^{-5}-10^{-4} \mathrm{erg} / \mathrm{s} / \mathrm{Hz}\right)$, where radio loud AGN deviate from the low luminosities correlation. X-ray emission in radio loud sources could have larger contributions from the jet.

optical and radio data. Our sample consists of all AGN (403 total, Figures 1 and 2) with available X-ray (2-10 keV), optical (B-band) and radio (5 GHz) data.

\section{First results and ongoing work}

Radio loud sources show jet contribution to optical and X-ray emission, and are brighter in X-rays than radio quiet. Optical and X-rays are AGN dominated with small contribution from host. For optical luminosities higher than $10^{-6} \mathrm{erg} / \mathrm{s} / \mathrm{Hz}$ radio loud AGN are brighter in X-rays than radio quiet. This effect increases for higher luminosities $\left(10^{-5}-10^{-4}\right)$, where loud AGN deviate from the low luminosities correlation. X-rays in radio loud sources could have higher contributions from the jet. The sample seems to be missing faint, radio loud AGN, although at this point it is not clear if this is due to selection or astrophysical effects.

While X-rays in radio loud AGN seem to come mainly from jets, other mechanisms of X-ray emission are being studied (e.g. ADAF)? A complete spectral optical and X-ray analysis, including also the $0.2-2 \mathrm{kev}$ band will bring light to the origin of the X-rays in these sources. We are currently studying the sample properties according to different classifications (Seyfert and QSO or FR I and FR II morphologies), and will include IR data when available, with the goal of carrying out a systematic analysis in as many wavelengths as possible.

\section{References}

Bianchi, S., Bonilla, N. F., Guainazzi, M., Matt, G., \& Ponti, G. 2009a, A\&̊A, 501, 915

Bianchi, S., Guainazzi, M., Matt, G., Fonseca Bonilla, N., \& Ponti, G. 2009b, A $\mathscr{E} A, 495,421$

Panessa, F., Barcons, X., \& Bassani L. et al., 2009, A\&A, 467, 519

Véron-Cetty, M. \& Véron, P. 2010, A\& A, 518, A10

Watson, M. G., Schröder, A. C., Fyfe, D., et al. 2009, A\&\&A, 493, 339 